\title{
ARTICLE
}

Received 27 Nov 2013 | Accepted 4 Jun 2014 | Published 30 Jun 2014

DOI: $10.1038 /$ ncomms5290

\section{Wigner and Kondo physics in quantum point contacts revealed by scanning gate microscopy}

\author{
B. Brun ${ }^{1,2}$, F. Martins ${ }^{3}$, S. Faniel ${ }^{3}$, B. Hackens ${ }^{3}$, G. Bachelier ${ }^{1,2}$, A. Cavanna ${ }^{4}$, C. Ulysse ${ }^{4}$, A. Ouerghi ${ }^{4}$, U. Gennser ${ }^{4}$, \\ D. Mailly ${ }^{4}$ S. Huant ${ }^{1,2}$, V. Bayot ${ }^{1,3}$, M. Sanquer ${ }^{1,5} \&$ H. Sellier ${ }^{1,2}$
}

Quantum point contacts exhibit mysterious conductance anomalies in addition to well-known conductance plateaus at multiples of $2 \mathrm{e}^{2} / \mathrm{h}$. These 0.7 and zero-bias anomalies have been intensively studied, but their microscopic origin in terms of many-body effects is still highly debated. Here we use the charged tip of a scanning gate microscope to tune in situ the electrostatic potential of the point contact. While sweeping the tip distance, we observe repetitive splittings of the zero-bias anomaly, correlated with simultaneous appearances of the 0.7 anomaly. We interpret this behaviour in terms of alternating equilibrium and nonequilibrium Kondo screenings of different spin states localized in the channel. These alternating Kondo effects point towards the presence of a Wigner crystal containing several charges with different parities. Indeed, simulations show that the electron density in the channel is low enough to reach one-dimensional Wigner crystallization over a size controlled by the tip position.

\footnotetext{
${ }^{1}$ University Grenoble Alpes, F-38000 Grenoble, France. ${ }^{2}$ CNRS, Inst. NEEL, F-38042 Grenoble, France. ${ }^{3}$ IMCN/NAPS, Université catholique de Louvain, B-1348 Louvain-la-Neuve, Belgium. ${ }^{4}$ CNRS, Laboratoire de Photonique et de Nanostructures, UPR20, F-91460 Marcoussis, France. ${ }^{5}$ CEA, INAC-SPSMS, F-38054 Grenoble, France. Correspondence and requests for materials should be addressed to H.S. (email: hermann.sellier@neel.cnrs.fr).
} 
Q uantum point contacts (QPCs) ${ }^{1}$ are among the simplest quantum devices made out of a two-dimensional electron gas (2DEG). Applying a negative voltage on a split-gate creates a quasi-one-dimensional (1D) channel connected to large $2 \mathrm{D}$ reservoirs. This narrow channel behaves as an electron waveguide and transmits a finite number of modes, each of them carrying one quantum of conductance $G_{0}=2 e^{2} / h$ ( $e$ is the electron charge and $h$ the Planck constant). As a result, the conductance versus gate voltage curve shows a series of quantized plateaus with transitions, which are well reproduced by a singleparticle model ${ }^{2}$.

However, since the early days of QPCs, a shoulder-like feature is commonly observed ${ }^{3}$ at a conductance around $0.7 G_{0}$, which cannot be explained by single-particle theories. With lowering temperature, this ' 0.7 anomaly' rises to reach the first plateau, and a zero-bias peak called 'zero-bias anomaly' (ZBA) emerges in the non-linear differential conductance ${ }^{4}$. These anomalies have been extensively studied through transport experiments ${ }^{3-7}$, revealing the complexity of the underlying phenomena. Different theoretical models have been proposed ${ }^{8-13}$, but no consensus could be reached so far on their interpretation ${ }^{14}$.

Recently, an experiment using several gates to vary the channel length ${ }^{15}$ revealed the possible existence of several emergent localized states responsible for the conductance anomalies. At the same time, a different theoretical model was proposed ${ }^{16}$, explaining the anomalies without invoking localized states in the channel. As stressed in ref. 17, investigating these anomalies using scanning probe techniques could make it possible to check the existence of spontaneously localized states and discriminate between these two proposals: this is the aim of the present letter.

Here we perform scanning gate microscopy (SGM) ${ }^{18}$, in which a negatively charged tip is scanned above the sample surface and modifies the electrostatic potential in the 2DEG. This local potential change induces electron backscattering towards the QPC, which can be used to image single-particle phenomena such as wave-function quantization in the channel ${ }^{19}$, branched flow in the disorder potential ${ }^{20}$, interference patterns induced by the tip $^{21-23}$, or to investigate electron-electron interactions inside ${ }^{24}$ or outside ${ }^{25}$ the QPC. This movable gate can also be used to tune in situ the saddle potential of the QPC, in a more flexible and less invasive way than fixed surface gates, and probe intrinsic properties of the QPC such as the 0.7 anomaly ${ }^{26,27}$.

Here we show that approaching the tip towards the QPC produces an oscillatory splitting of the ZBA, correlated with simultaneous appearances of the 0.7 anomaly, thereby confirming that both features share a common origin ${ }^{4,15}$. We interpret these observations as the signature of a small one-dimensional Wigner crystal $^{28-30}$ forming in the channel ${ }^{31}$ (a quantum chain of charges localized by Coulomb interactions in absence of disorder). The number of charges in this many-body correlated state is tuned by changing the tip position, leading alternatively to a single- or a two-impurity Kondo effect (screening of a localized spin by conducting electrons), with a conductance peak either at zero, or at finite bias, depending on the charge parity.

Our observations therefore strongly support the existence of emergent localized states, as suggested in ref. 15 where the number of localized charges is controlled by changing the effective channel length. Here we show that a similar effect is observed when changing the distance of an additional gate placed around the QPC. To understand this new result, we perform classical electrostatic simulations and evaluate the size of the region where electrons should form a $1 \mathrm{D}$ Wigner crystal, thanks to the critically low electron density. We show that the calculated size of this small crystal is in good agreement with the observed change in the number of localized charges, thereby revealing that Wigner crystallization is, to our opinion, the correct way to understand this spontaneous localization.

\section{Results}

Transport measurements. The QPC (see Methods and Fig. 1a) is cooled down to a temperature of $20 \mathrm{mK}$ in a cryogenic scanning probe microscope $\mathrm{e}^{32}$. In the absence of the tip (moved several microns away), the linear conductance shows the usual staircase behaviour versus gate voltage (Fig. 1b). The shoulder below the first quantized plateau is the puzzling 0.7 anomaly. The sourcedrain bias spectroscopy (Fig. 1c) shows that this shoulder evolves to a clear plateau at $0.85 G_{0}$ at finite bias $5,33,34$. The narrow peak around zero bias is the ZBA and disappears above $1 \mathrm{~K}$ (Supplementary Fig. 1). Its width of $200 \mu \mathrm{eV}$ is much smaller than the $1 \mathrm{D}$ subband spacing of $4.5 \mathrm{meV}$ (Fig. 1d). Above $0.7 G_{0}$, the ZBA splits into finite-bias peaks ${ }^{15,35}$ centred at $\pm 250 \mu \mathrm{V}$. We show in the following that the presence of the 0.7 anomaly is related to this splitting of the $\mathrm{ZBA}$.

Scanning gate microscopy. When the tip is scanned near the QPC and polarized such as to deplete locally the 2DEG (see Methods), we observe two distinct phenomena. On the first
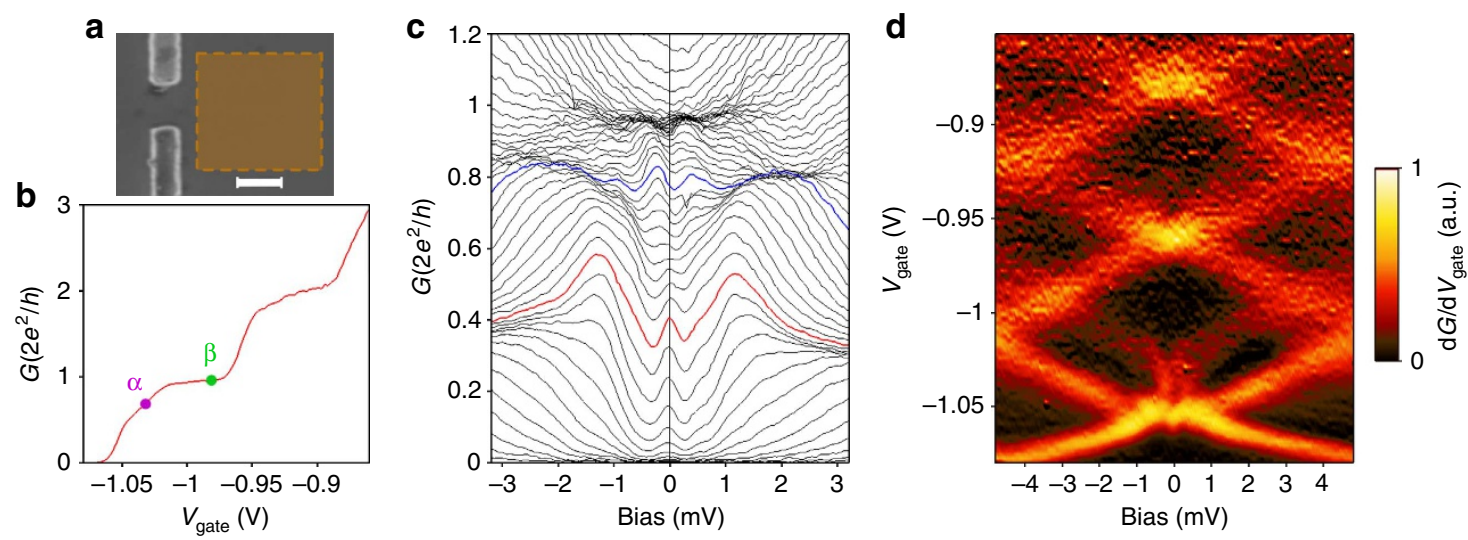

Figure 1 | Transport measurements. Base temperature is $20 \mathrm{mK}$. (a) Electron micrograph of the QPC gates. Scale bar, $300 \mathrm{~nm}$. The dashed box indicates the position of the scanning area used in Fig. 2. (b) Differential conductance $G$ at zero bias versus split-gate voltage $V_{\text {gate }}$. The 0.7 anomaly is visible below the first plateau. Positions $\alpha$ and $\beta$ are used in Fig. 2a,b. (c) Differential conductance $G$ versus source-drain bias for different gate voltage $V_{\text {gate }}$ from -1.08 to $-0.96 \mathrm{~V}$. The zero-bias peak in the red curve splits into finite-bias peaks in the blue curve. (d) Numerical derivative of the differential conductance $\mathrm{d} G / \mathrm{d} V_{\text {gate }}$ versus bias and gate voltage. Yellow lines highlight transitions between conductance plateaus. 

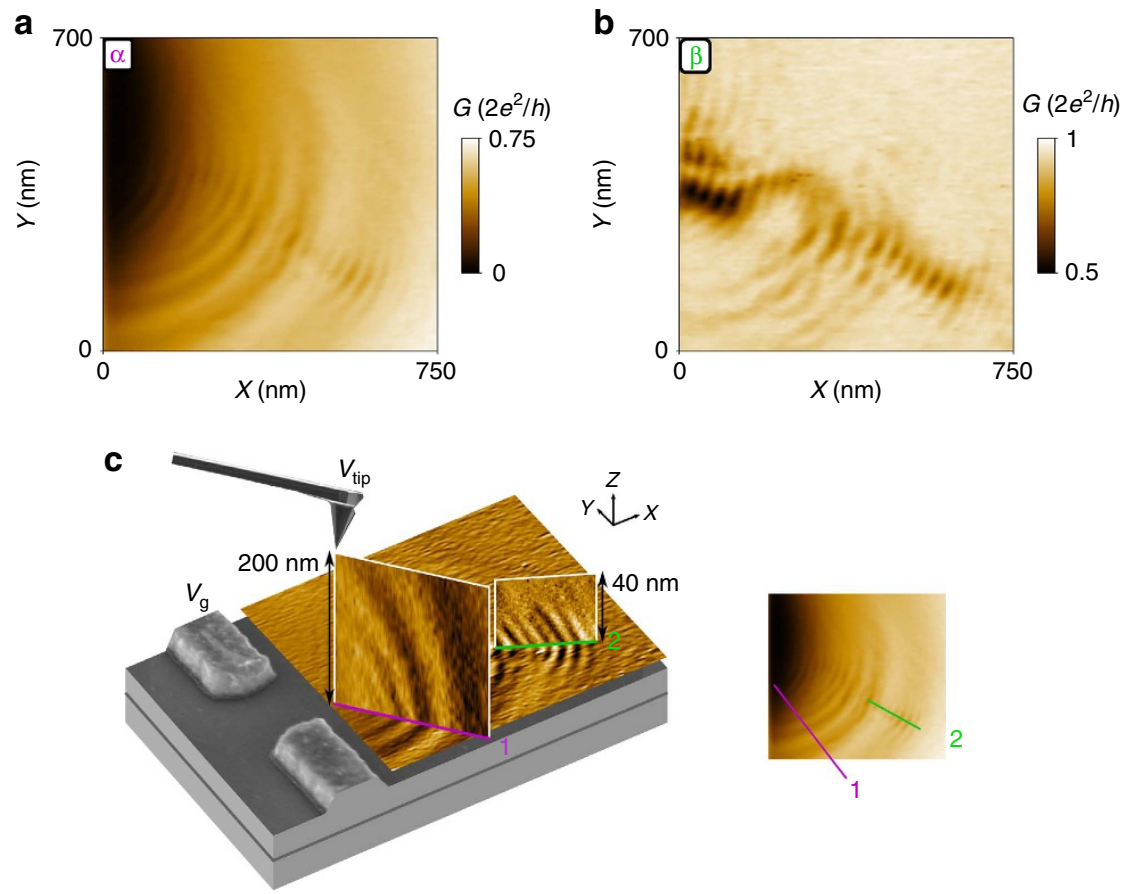

Figure 2 | Scanning gate microscopy. Base temperature is $20 \mathrm{mK}$. (a,b) SGM maps of the QPC conductance $G$ versus tip position in the (X,Y) horizontal plane for gate voltages $V_{\text {gate }}=-1 \mathrm{~V}(\mathbf{a})$ and $-0.95 \mathrm{~V}(\mathbf{b})$ corresponding, respectively, to points $\alpha$ and $\beta$ as defined in Fig. $1 \mathrm{~b}$ (gate voltages are shifted by $35 \mathrm{mV}$ in presence of the tip). Concentric rings are only visible at $\alpha$, and interference fringes are more contrasted at $\beta$. Additional data are presented in Supplementary Fig. 2. (c) Schematic view of the SGM experiment showing the tip scanning above the 2DEG near the QPC gates and three SGM maps. The horizontal map is the same as in $\mathbf{a}$, but the data have been differentiated with respect to the Y-coordinate to highlight details. The two vertical maps are recorded in planes perpendicular to the surface along the purple line 1 (size $500 \times 200 \mathrm{~nm}$, gate voltage $\alpha$ ) and the green line 2 (size $250 \times 40 \mathrm{~nm}$, gate voltage $\beta$ ) as indicated on the right image (identical to $\mathbf{a}$ ). The two vertical maps have been differentiated with respect to their horizontal coordinate to highlight details (raw data are shown in Supplementary Fig. 3). The vertical map along line 1 reveals that the concentric rings visible in a form also rings in the vertical plane, whereas the vertical map along line 2 shows that interference fringes disappear rapidly with the tip-to-surface distance.

conductance plateau (Fig. 2b), SGM images reveal the electron flow coming out of the QPC, with fringes spaced by half the Fermi wavelength, as already observed by several group ${ }^{20,22,23}$. The fringes result from interferences of electrons backscattered by the depleted region below the tip and reflected by impurities ${ }^{20,22}$ in the $2 \mathrm{DEG}$ or directly by the gates ${ }^{21,36}$.

Below the first plateau (Fig. 2a), SGM maps reveal a novel set of concentric rings centred on the QPC, with a spacing increasing with tip distance (see also Supplementary Fig. 2). As opposed to the previous one-particle interference fringes, these new rings are not linked to the electron flow (black region in Fig. 2b) but extend rather isotropically around the QPC, not only in the horizontal plane but in all three directions of space. This is revealed by scanning the tip in a vertical plane (Fig. 2c), unveiling half spheres centred on the QPC (purple line 1). This behaviour contrasts with that of interference fringes (green line 2) that quickly disappear when the tip is scanned $>50 \mathrm{~nm}$ above the surface (see also Supplementary Fig. 3). Interferences indeed require electrons at the Fermi level to be backscattered by a depleted region below the tip, a situation which is only obtained for the tip close enough to the 2DEG (and at low enough temperature to avoid thermal averaging of the interferences). We therefore conclude that the new rings are not interferences but result from a direct tuning of the electrostatic potential in the QPC. The larger ring spacing at larger distances results from the smaller potential changes induced by the tip.

Conductance anomalies. To demonstrate that these rings correspond to modulations of the conductance anomalies, the tip is scanned along a single line in a region with almost no interference (line 3 in Fig. 3a) and the QPC parameters (gate and bias voltages) are varied. Figure $3 \mathrm{~b}$ shows that the ring-related conductance oscillations are only visible for gate voltages in the transition below the first plateau, just where the ZBA and 0.7 anomaly are observed. Figure $3 c$ shows how the conductance oscillations evolve when the average conductance goes from 0 to $G_{0}$ while changing the gate voltage. The oscillations are clearly visible between 0.4 and $0.8 G_{0}$. They are blurred when approaching $G_{0}$ because some interference fringes come into play. The increasing distance between conductance extrema (labelled A to $\mathrm{D}$ for maxima and A' to $\mathrm{D}^{\prime}$ for minima) is consistent with an oscillatory phenomenon in the QPC, controlled by the decreasing electrostatic coupling to the tip. Plotting the conductance versus gate voltage (Fig. 3d) reveals the oscillatory behaviour of the 0.7 anomaly. The amplitude of this modulation can be read from Fig. 3e, where curves at positions $\mathrm{X}$ and $\mathrm{X}^{\prime}$ are compared two-bytwo (curves are shifted horizontally to compensate for the drift of the pinch-off voltage while approaching the tip). Curves at positions $\mathrm{A}$ to $\mathrm{D}$ are smooth with no shoulder, that is, no anomaly, whereas curves at positions $\mathrm{A}^{\prime}$ to $\mathrm{D}^{\prime}$ present a reduced conductance above $0.5 G_{0}$, that is, the 0.7 anomaly. The concentric rings observed in SGM images (Fig. 2a) therefore correspond to an alternating modulation of the 0.7 anomaly when the tip approaches the QPC.

We now analyse the behaviour of the ZBA when the 0.7 anomaly repeatedly appears and disappears, and show that both anomalies are linked. Figure $4 \mathrm{a}$ shows the differential conductance versus source-drain bias for different tip positions (same scan line as in Fig. 3a). Curves at positions A to D have a peak 
a
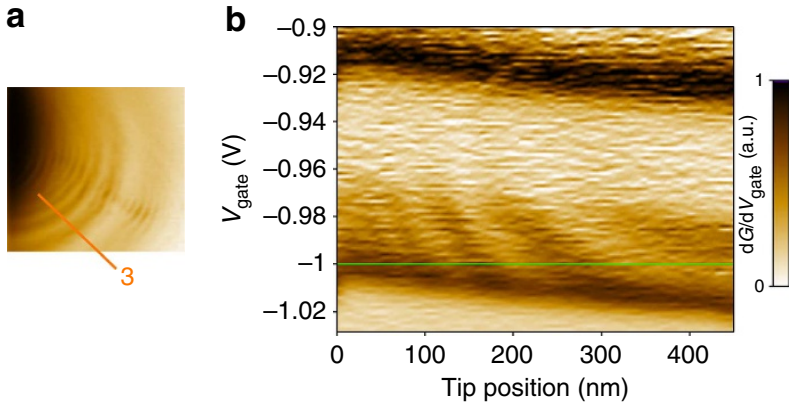

d

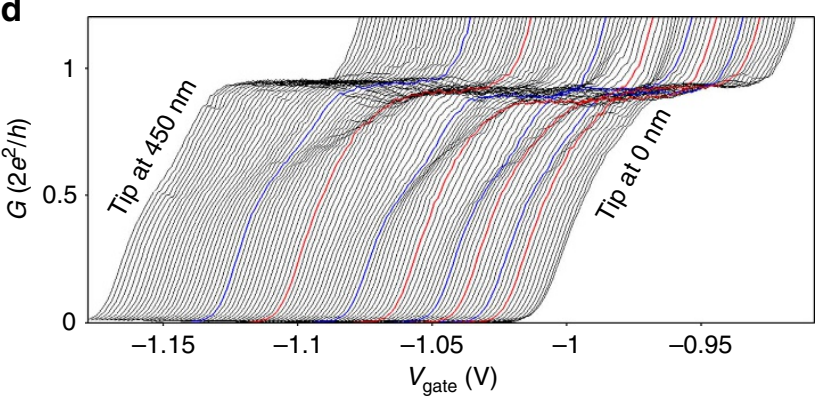

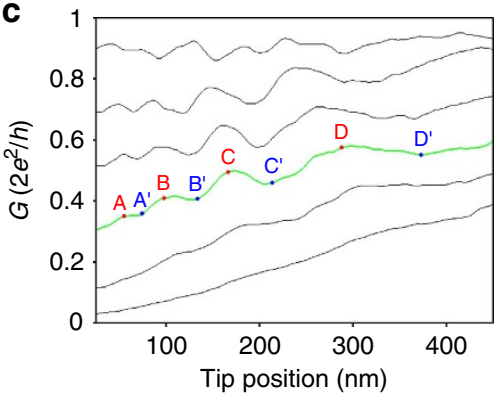

e

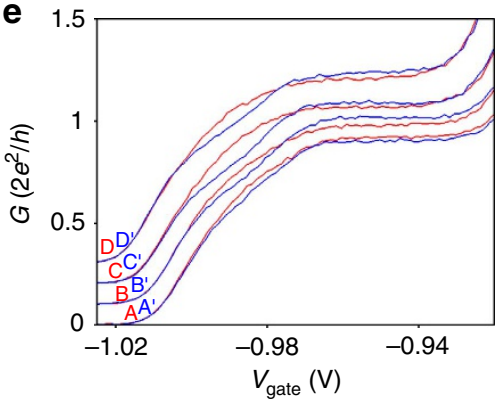

Figure 3 | Modulation of the $\mathbf{0 . 7}$ anomaly. The figure analyses the zero-bias conductance oscillations when the tip is scanned along the orange line 3 indicated in a with the origin of positions in the QPC direction. (b) Trans-conductance $\mathrm{dG} / \mathrm{d} V_{\text {gate }}$ versus tip position and gate voltage. Black regions correspond to transitions between plateaus. The conductance oscillations are only visible below the first plateau. (c) Conductance $G$ versus tip position for gate voltages $V_{\text {gate }}=-0.964,-0.983,-0.992,-1.000,-1.006$ and $-1.012 \mathrm{~V}$ (from top to bottom). Conductance extrema at $V_{\text {gate }}=-1 \mathrm{~V}$ (green curve) are labelled $A$ to $D$ (maxima) and $A^{\prime}$ to $D^{\prime}$ (minima). The global slope corresponds to the rise of the saddle-point potential when the tip approaches the QPC. (d) Conductance $G$ versus gate voltage for different tip positions from 0 to $450 \mathrm{~nm}$ (successive curves are shifted to the left). (e) Same data as in $\mathbf{d}$ but for tip positions $A$ to $D$ (red curves, shifted vertically) and $A^{\prime}$ to $D^{\prime}$ (blue curves, shifted also horizontally to be compared with red curves). Red curves show no shoulder, whereas blue curves show the 0.7 anomaly. Small differences between plateau values come from residual interference fringes.

centred at zero bias (ZBA), whereas curves at positions $\mathrm{A}^{\prime}$ to $\mathrm{D}^{\prime}$ have a dip at zero bias and local maxima at $\pm 250 \mu \mathrm{V}$ bias (splitting of the ZBA), on top of the same V-shaped background. Scanning the SGM tip therefore produces a repetitive splitting of the ZBA, which draws a checkerboard pattern in a colour plot of the spectroscopy versus tip position (Fig. 4b). Note that the spontaneous splitting of the $\mathrm{ZBA}$ observed without the tip (Fig. 1c) also shows peaks at $\pm 250 \mu \mathrm{V}$ and probably has the same origin.

Considering the regularity of the concentric rings in Fig. 2a, this oscillatory behaviour of the 0.7 and ZBAs would be observed for any scanning line in a large range of angles (see Supplementary Fig. 4 and Supplementary Note 1). As a consequence, rings with conductance maxima correspond to a simple staircase in the linear conductance and a ZBA in the non-linear spectroscopy, whereas rings with conductance minima correspond to a 0.7 anomaly and a splitting of the ZBA. This result shows that the ZBA suppresses the 0.7 anomaly at low temperature ${ }^{4}$ only if the ZBA is not split into finite-bias peaks.

\section{Discussion}

First, we would like to stress again that these new conductance oscillations cannot be explained by interference effects in the 2DEG. One argument already given above is that interferences require backscattering with a tip close to the surface, whereas the new rings are observed up to large tip heights (Fig. 2c). A second argument is that interference fringes would have an increasing spacing for short tip distances because the density is reduced close to the QPC and the electron wavelength is larger, but the opposite behaviour is observed.
We now discuss a possible single-particle effect inside the QPC that, at first sight, could give similar conductance oscillations. In case of a non-adiabatic transmission, wave functions are scattered by the QPC potential barrier and transmission resonances appear when the barrier length is equal to an integer number of half the longitudinal wavelength. If the effect of the tip is to change the channel length, such resonances could give conductance oscillations versus tip distance. However, this single-particle mechanism cannot explain the repetitive splittings of the ZBA, which are simultaneous with the observed conductance oscillations, and we therefore need another explanation.

The ZBA in QPCs has been shown to scale with temperature and magnetic field similar to the Kondo effect in quantum dots ${ }^{4}$. This effect corresponds to the screening of a single degenerate level by a continuum of states, and therefore indicates the presence of a localized spin in the QPC channel ${ }^{11}$. Splittings of the ZBA have been observed recently in length-tunable QPCs ${ }^{15}$ and interpreted as a two-impurity Kondo effect ${ }^{37,38}$, involving non-equilibrium Kondo screening ${ }^{39,40}$, as commonly observed in quantum dots with even numbers of electrons ${ }^{41}$, coupled quantum dots ${ }^{42}$ and molecular junctions ${ }^{43}$.

We now consider different scenarios to explain the presence of such localized states in our system. In a recent work on QPCs made out of a 2D hole gas, a spontaneous splitting of the ZBA as the QPC opens has been reported ${ }^{35}$. This effect was attributed to a charge impurity forming a potential well close to the channel, containing one or two charges, leading to different types of Kondo screening. In our case, the spontaneous splitting of the ZBA as the QPC opens (Fig. 1c) could be explained by this effect. However, the fact that approaching the tip towards the QPC results in four successive splittings of the ZBA indicates that this impurity should contain at least eight charges, which is unlikely 


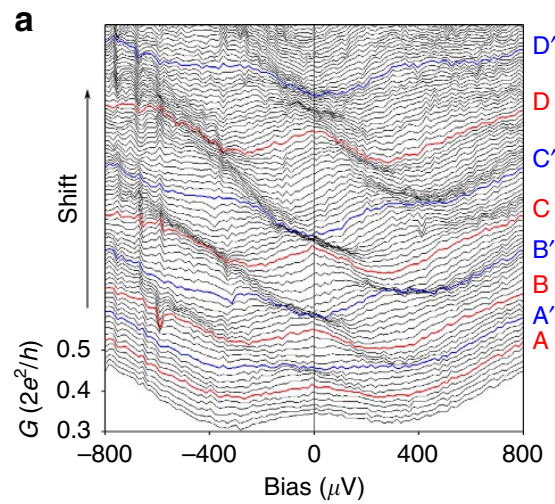

C
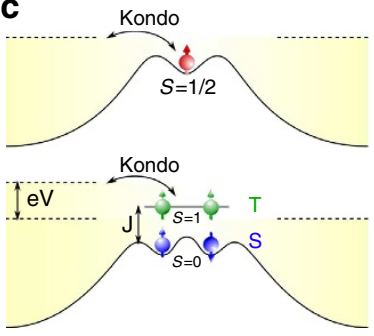
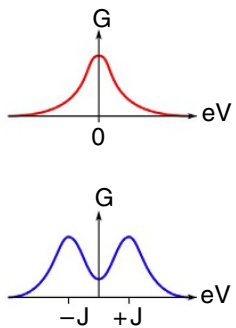

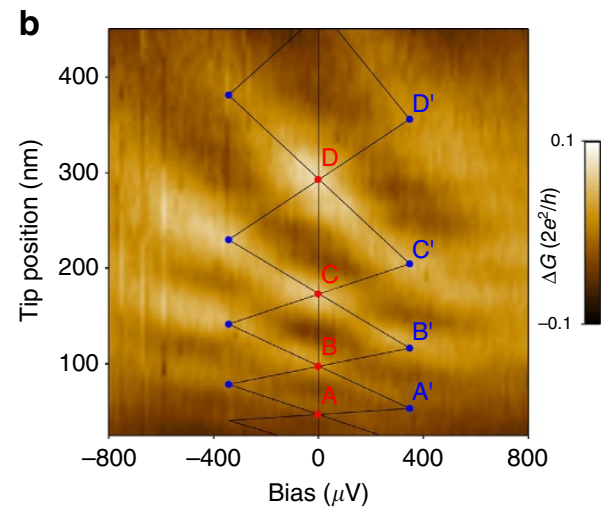

d

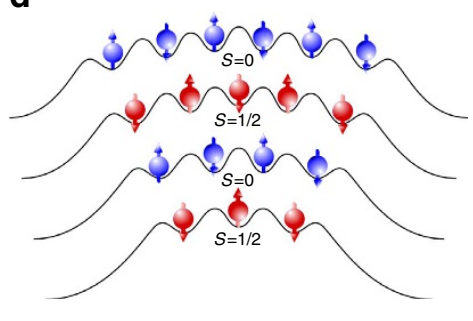

Figure 4 | Successive splittings of the ZBA. The figure analyses the low bias source-drain spectroscopy when the tip is scanned along the orange line 3 indicated in Fig. 3a. (a) Differential conductance $G$ versus source-drain bias at a fixed gate voltage $V_{\text {gate }}=-1 \mathrm{~V}$ for different tip positions from 0 to $450 \mathrm{~nm}$. Successive curves are shifted upwards by $0.0075 \times 2 \mathrm{e}^{2} / \mathrm{h}$. Conductance peaks are visible at zero and finite bias on red and blue curves, respectively. (b) Colour plot of the same data as in $\mathbf{a}$ after subtraction of a smooth background to suppress the main gating effect of the tip. Peak positions are indicated by dots. The successive ZBA splittings give a checkerboard pattern. The asymmetry results from the bias-induced change of the QPC position. (c) Schematic of the QPC potential with one (top) and two (bottom) localized electrons, corresponding, respectively, to a $S=1 / 2$ ground state with a zerobias Kondo peak and to a $S=0$ ground state with finite-bias Kondo peaks involving the excited state $S=1$ with singlet-triplet energy splitting $J$. The expected conductance $G$ versus bias $V$ is shown on the right for each state. (d) Schematic of the QPC potential with an increasing number of electrons localized by Coulomb interactions. The antiferromagnetic spin coupling in this small 1D Wigner crystal gives either a $S=1 / 2$ ground state $(Z B A)$ or a $S=0$ ground state (splitting of the ZBA), depending on the parity (respectively odd or even).

for a single impurity. Nevertheless, one could imagine that a shallow quantum dot has formed in the QPC due to potential fluctuations induced by residual disorder ${ }^{44}$ and giving Coulomb blockade oscillations as often observed in long $1 \mathrm{D}$ wires ${ }^{45}$. The major argument to exclude this scenario is that the split-gate has a larger capacitive coupling to the channel than the tip has (that is, a larger lever-arm parameter), so the split-gate should induce more charging events than the tip, but we observe the opposite: approaching the tip by $600 \mathrm{~nm}$ produces four successive splittings of the ZBA and sweeping the gate voltage produces only one splitting. It can therefore not be Coulomb blockade in a disorder-induced quantum dot.

The only remaining possibility to explain the presence of localized states in the channel is a spontaneous electron localization, which is not induced by potential barriers but instead by electron-electron interactions. Indeed, a large number of theoretical and numerical investigations show that interactions can localize a finite number of electrons in the channel ${ }^{12,13,46,47}$. On the first conductance plateau and below, transport can be considered as 1D, and the electron density is so low that the Coulomb repulsion overcomes the kinetic energy. When the $1 \mathrm{D}$ density $n_{1 \mathrm{D}}$ fulfills the criterion $n_{1 D} \times a_{\mathrm{B}}<1$, where $a_{\mathrm{B}}$ is the effective Bohr radius ( $10 \mathrm{~nm}$ in GaAs), electrons are expected to spontaneously order in a crystal, with an interparticle distance minimizing Coulomb repulsion ${ }^{48}$. This many-body state, known as a Wigner crystal $^{28,29}$, has been suggested to be responsible for the 0.7 anomaly in QPCs ${ }^{12}$. When the electron density in the channel is decreased below the critical value, the density modulations evolve continuously from the $\lambda_{\mathrm{F}} / 2$ periodicity of Friedel oscillations to the $\lambda_{\mathrm{F}} / 4$ periodicity of the Wigner crystal $^{49}$. Quantum Monte Carlo simulations have also shown that electrons in the crystallized region can be relatively decoupled from the high-density reservoirs and present an antiferromagnetic coupling $J$ between adjacent spins ${ }^{47}$. In contrast to the case of quantum dots with real tunnel barriers, electron localization in a QPC is not straightforward and results from emergent barriers in the self-consistent potential. On the other hand, the Kondo effect requires a relatively open system with a good coupling to the reservoirs, and this makes the QPC a suitable platform to observe Kondo phenomena on an interaction-induced localized state, as shown recently in lengthtunable QPCs ${ }^{15}$.

This last scenario being the most realistic one in our case, we therefore interpret the four observed oscillations as a signature of eight successive states of a small non-uniform 1D Wigner crystal with an alternating odd and even number of localized charges. Situations with an odd number of electrons in a spin $S=1 / 2$ ground state show a ZBA due to Kondo screening of non-zero spin states. Situations with an even number of electrons in a spin singlet $S=0$ ground state show a splitting of the ZBA due to nonequilibrium Kondo screening 39,40 of the spin triplet $S=1$ excited state with peaks at a finite bias $e V=J$ (Fig. 4c). The four oscillations, suggestive of eight successive states, reveal that a large number of electrons can spontaneously localize in the channel of a QPC, as shown in Fig. 4d. Observing Kondo screening on a system with many localized charges is not so surprising if we compare with quantum dots where the Kondo effect is observed up to large numbers of electrons ${ }^{50}$. 
Nevertheless, the particular case of a 1D chain of localized charges in the Kondo regime still requires theoretical investigations.

This analysis is consistent with the interpretation given in ref. 15 for similar observations using a QPC with six surface gates to tune the channel length. Our SGM experiment brings additional information on this effect, as scanning the tip around the QPC, laterally or vertically, changes the shape, extension and symmetry of the channel potential. The circular and almost isotropic rings in Fig. $2 \mathrm{c}$ show that the localized states survive to all these potential deformations. The regularity of the successive rings also suggests that this localization occurs rather independently of disorder, although possible crystal pinning effects should be investigated in the future.

In ref. 15, the parameter controlling the number of localized states is the effective length of the channel, defined in ref. 51 and computed using an analytical approach assuming a fixed zero potential at the surface ${ }^{52}$. This method is not suitable to model our SGM experiment, as the tip is situated above the surface. To evaluate the potential landscape in the presence of the tip, we perform 3D classical electrostatic simulations in the ThomasFermi approximation (see Methods and Supplementary Note 2) and compute self-consistently the local potential $V(x, y)$ in the $2 \mathrm{DEG}$ and the local $2 \mathrm{D}$ electronic density $n_{2 \mathrm{D}}(x, y)$ (Fig. 5a). In this way, the tip-induced potential is correctly calculated, with the screening effects from the 2DEG and the metallic gates taken into account. We obtain a good agreement between calculated and experimental values regarding the gate voltage required to close the QPC, the tip voltage to reach depletion in the 2DEG and the cross-talk between the tip position and the QPC opening. The effective channel length used in ref. 15 was calculated in ref. 51, using the unscreened gate potential. This length cannot be calculated here from our self-consistent potential, because screening effects induce non-parabolic transverse confinement potentials.

We propose instead that the parameter controlling the number of localized charges is the size of the region where the 1D Wigner crystallization should occur. This interaction-induced spontaneous ordering is often discussed in terms of the Wigner-Seitz radius $r_{\mathrm{s}}=1 /\left(2 n_{1 \mathrm{D}} a_{\mathrm{B}}\right)$, representing the ratio of the Coulomb repulsion to the kinetic energy. A recent numerical investigation of the 1D Wigner crystallization shows that the critical parameter $r_{\mathrm{s}}^{\mathrm{c}}$ varies between 0.5 and 2, depending on the strength of the transverse confinement potential ${ }^{48}$. To evaluate the size of the

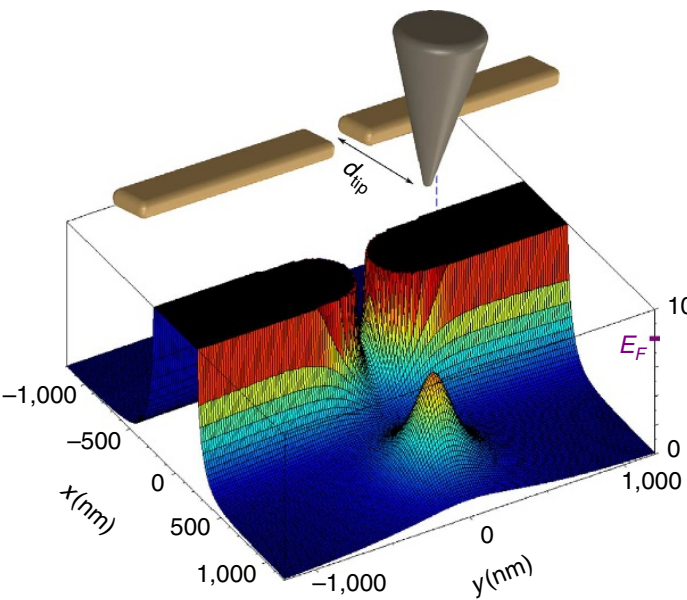

d

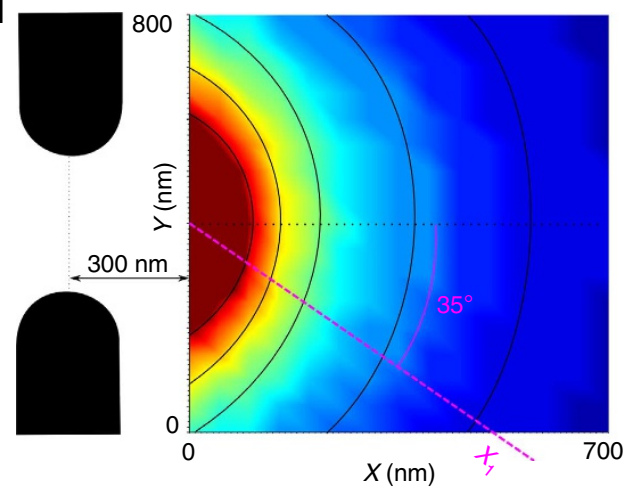

b
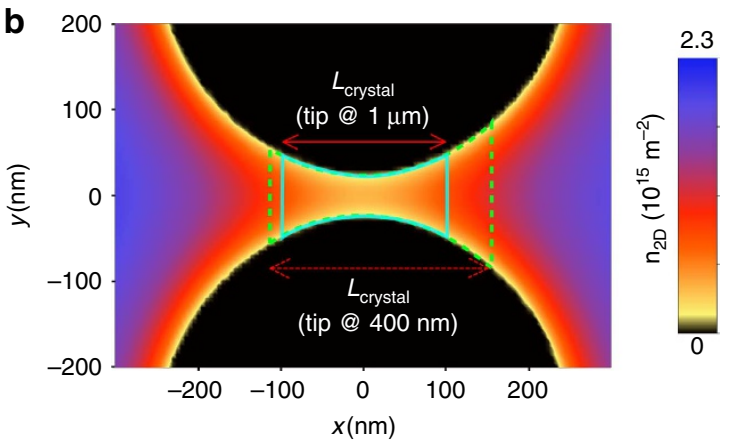

C
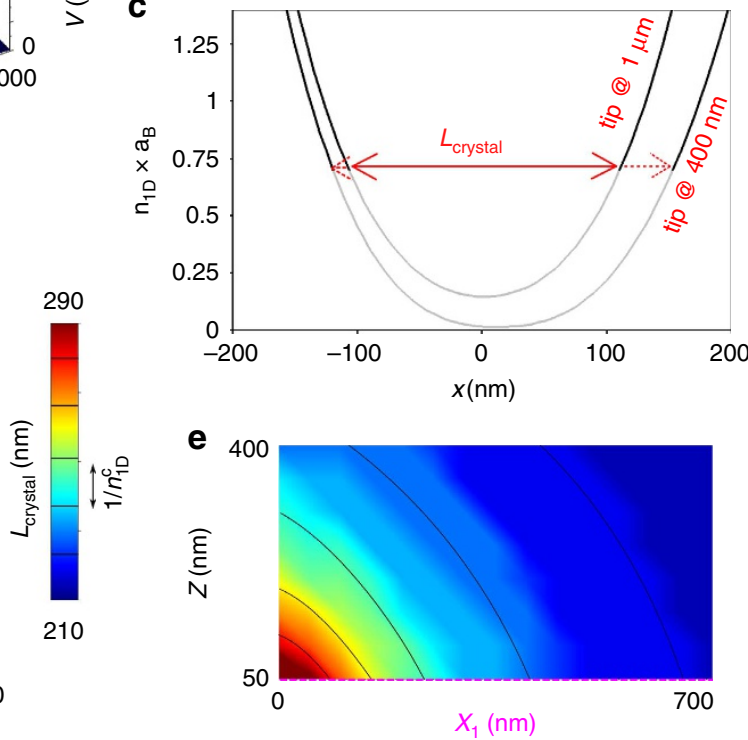

Figure 5 | Calculation of the electron density and estimation of the Wigner crystal size. (a) Geometry of the metallic gates and SGM tip defined in the Comsol simulation software and example of electrostatic potential map computed for a given gate voltage and tip position. The Fermi energy $E_{\mathrm{F}}$ is $8 \mathrm{meV}$ in the 2DEG. (b) Map of the two-dimensional electron density $n_{2 D}$ in the 2DEG computed classically but self-consistently with the potential, when the tip is at $1 \mu \mathrm{m}$ from the QPC. (c) One-dimensional electron density $n_{1 \mathrm{D}}$ obtained by integration of $n_{2 \mathrm{D}}$ along the $y$ axis, when the tip is at $1 \mu \mathrm{m}$ and $400 \mathrm{~nm}$ from the QPC. Choosing a critical density $n_{1 \mathrm{D}}^{\mathrm{c}}=0.7 / a_{\mathrm{B}}$ determines the expected size $L_{\text {crystal }}$ of the $1 \mathrm{D}$ Wigner crystal. (d) Computed size of the Wigner crystal as a function of tip position in an horizontal plane $30 \mathrm{~nm}$ above the surface $\left(V_{\text {gate }}=-1 \mathrm{~V}\right)$. The region in red corresponds to a closed contact (the electron density is zero at the QPC center for these tip positions). Black lines indicate tip positions for which $L_{\text {crystal }}$ is enlarged by $1 / n_{1 D}^{c}$ corresponding at first order to the addition of one charge to the crystal. (e) Computed size of the Wigner crystal for tip positions in a vertical plane (above line $X_{1}$ at $35^{\circ}$ from QPC axis). 
region where $r_{\mathrm{s}}$ is larger than a given threshold, we calculate the $1 \mathrm{D}$ electron density by integration of the $2 \mathrm{D}$ electron density in the transverse direction (Fig. 5b,c). As an example, we choose a critical value $r_{\mathrm{s}}^{\mathrm{c}}=0.71$ corresponding to a critical density $n_{1 \mathrm{D}}^{\mathrm{c}}=0.7 / a_{\mathrm{B}}$, and evaluate the size $L_{\text {crystal }}$ where the density is lower than $n_{\mathrm{lD}}^{\mathrm{c}}$. This size is found to vary from 210 to $290 \mathrm{~nm}$ when the tip is approached by $600 \mathrm{~nm}$ towards the QPC, which shows that the tip can strongly affect the size of the low-density region, and hence the number of localized charges. The tip positions leading to the same $L_{\text {crystal }}$ form rings centred on the QPC, both for horizontal and vertical scanning planes (Fig. 5d,e), in the same way as the conductance oscillations observed in the SGM experiment (Fig. 2c).

Our classical simulation holds only for an estimate of the size $L_{\text {crystal }}$, but cannot be used to calculate the number of localized charges, as quantum mechanics dominates at such a low density. Note that charges in this crystal are not expected to be uniformly spaced, because the potential of a QPC shows a strong curvature. This non-uniform situation would require an extension of the concept of Wigner crystal, which is usually studied in a flat potential landscape. A rather crude approach to evaluate how many charges can be added by approaching the tip is to suppose that one charge is added to the crystal each time the region is enlarged by $1 / n_{1 \mathrm{D}}^{\mathrm{c}}\left(\sim 14 \mathrm{~nm}\right.$ for $\left.r_{\mathrm{s}}^{\mathrm{c}}=0.71\right)$. With this assumption, approximately five charges can be added to the crystal when the tip is approached close to the QPC (Fig. 5d). This value is qualitatively consistent with the four oscillations observed in the experiment, and interpreted as the addition of eight charges. Simulations also show that the number of charges can be modified simply by changing the split-gate voltage (see Supplementary Fig. 5). This could explain the ZBA splitting observed above $0.7 G_{0}$ in absence of the tip (Fig. 1c).

Our assumption that electrons form a $1 \mathrm{D}$ system in the lowdensity region is justified $a$ posteriori by the fact that only the first and second transverse modes are occupied over the length $L_{\text {crystal }}$. The presence of the second mode at the extremities of this region indicates that the system is not strictly $1 \mathrm{D}$, but theory still predicts the formation of a Wigner crystal in the second sub-band of quasi-1D wires, forming a zigzag chain ${ }^{53}$, as possibly observed in experiment ${ }^{31,54}$. Interestingly, the simulations show that a small crystallized region survives when the second mode reaches the central part of the channel, which could explain the 0.7 analogues often observed between the first and second conductance plateaus.

In summary, we observe a periodic modulation of the conductance anomalies in a QPC at very low temperature while tuning continuously the potential with the polarized tip of a SGM. We explain this experimental observation by the formation of an interaction-induced localized state in the QPC channel, which gives rise to a single- or two-impurity Kondo effect depending on the odd or even number of localized charges, respectively. Indeed, electrostatic simulations show that the electron density in the channel is low enough to result in a spontaneous 1D Wigner crystallization. Our study gives new information on QPC conductance anomalies, which should guide future theoretical works, and will open the way to further experimental investigations involving fine tuning of the QPC potential using various methods.

\footnotetext{
Methods

Sample and measurement. The QPC is designed on a GaAs/AlGaAs heterostructure hosting a 2DEG $105 \mathrm{~nm}$ below the surface with $2.5 \times 10^{11} \mathrm{~cm}^{-2}$ electron density and $1.0 \times 10^{6} \mathrm{~cm}^{2} \mathrm{~V}^{-1} \mathrm{~s}^{-1}$ electron mobility. A Ti/Au split-gate is defined by e-beam lithography on a mesa with four ohmic contacts and forms a $270-\mathrm{nm}$ long and 300-nm-wide opening. The device is fixed to the mixing chamber of a dilution fridge, in front of a cryogenic scanning probe microscope ${ }^{32,55,56}$. The QPC is cooled down to a base temperature of $20 \mathrm{mK}$ at zero gate voltage. The four-probe
}

differential conductance $G=\mathrm{d} I / \mathrm{d} V_{\text {bias }}$ is measured by a standard lock-in technique, using a $10-\mu \mathrm{V}$ AC excitation at a frequency of $123 \mathrm{~Hz}$. A series resistance of $600 \Omega$ is subtracted from all data to have the conductance of the first plateau at $2 e^{2} / h$. As the temperature evolution of the zero-bias peak does not saturate below $90 \mathrm{mK}$, the temperature of electrons in the QPC is probably below this value.

Scanning gate microscopy. The tip of a commercial platinum-coated cantilever is fixed on a quartz tuning fork, which is mounted on the microscope actuators. The position of the QPC is determined by SGM as the tip position corresponding to the maximum change in conductance while scanning at large tip-surface distance. Next, the tip is lowered to a few tens of nanometres above the surface and scanned at fixed height on a single side of the 200-nm-thick split-gate in the scanning area shown in Fig. 1a. All the SGM results reported here are obtained for a tip voltage of $-6 \mathrm{~V}$ and a tip-to-surface height of $40 \mathrm{~nm}$ (except for vertical scans in Fig. 2c starting at $30 \mathrm{~nm}$ ). Note that the dilution fridge stays at its base temperature of $20 \mathrm{mK}$ during tip scanning.

Electrostatic simulations. Classical electrostatic simulations are performed with the Comsol software. We model the system in three dimensions as follows. The 2DEG plane is located $105 \mathrm{~nm}$ below the surface according to our heterostructure. The region between the 2DEG and the surface is filled with the GaAs dielectric constant $\epsilon_{r}=12.9$. The initial electron density in the 2DEG is set at $2.5 \times$ $10^{11} \mathrm{e}^{-} \mathrm{cm}^{-2}$ by the addition of a uniform plane of positive charges modelling ionized dopants (in the same plane as the 2DEG for better computation stability) The metallic gates are $120 \mathrm{~nm}$ thick and define a $270-\mathrm{nm}$-wide and $300-\mathrm{nm}$-long constriction, corresponding to our sample geometry. The tip is modelled by a cone with a $30^{\circ}$ full angle and a $30-\mathrm{nm}$ curvature radius at the apex. The tip voltage is fixed at $-6 \mathrm{~V}$ as in the experiment. For a given choice of gate voltage and tip position, the local potential and density are computed self-consistently by successive iterations. These calculations therefore include screening effects in the 2DEG.

\section{References}

1. van Wees, B. J. et al. Quantized conductance of point contacts in a twodimensional electron gas. Phys. Rev. Lett. 60, 848 (1988).

2. Büttiker, M. Quantized transmission of a saddle point constriction. Phys. Rev. B 41, 7906 (1990).

3. Thomas, K. J. et al. Possible spin polarization in a one-dimensional electron gas. Phys. Rev. Lett. 77, 1 (1996).

4. Cronenwett, S. M. et al. Low-temperature fate of the 0.7 structure in a point contact: a Kondo-like correlated state in an open system. Phys. Rev. Lett. 88, 226805 (2002).

5. Kristensen, A. et al. Bias and temperature dependence of the 0.7 conductance anomaly in quantum point contacts. Phys. Rev. B 62, 16 (2000).

6. Reilly, D. J. et al. Density-dependent spin polarization in ultra-low-disorder quantum wires. Phys. Rev. Lett. 89, 246801 (2002).

7. Hew, W. K. et al. Spin-incoherent transport in quantum wires. Phys. Rev. Lett 101, 036801 (2008).

8. Wang, C.-K. \& Berggren, K.-F. Local spin polarization in ballistic quantum point contacts. Phys. Rev. B 57, 4552 (1998).

9. Spivak, B. \& Zhou, F. Ferromagnetic correlations in quasi-one-dimensional conducting channels. Phys. Rev. B 61, 16730 (2000).

10. Sushkov, O. P. Conductance anomalies in a one-dimensional quantum contact. Phys. Rev. B 64, 155319 (2001).

11. Meir, Y., Hirose, K. \& Wingreen, N. S. Kondo model for the 0.7 anomaly in transport through a quantum point contact. Phys. Rev. Lett. 89, 196802 (2002)

12. Matveev, K. A. Conductance of a quantum wire in the Wigner-crystal regime. Phys. Rev. Lett. 92, 106801 (2004).

13. Rejec, T. \& Meir, Y. Magnetic impurity formation in quantum point contacts. Nature 442, 900 (2006).

14. Micolich, A. P. What lurks below the last plateau: experimental studies of the $0.7 \times 2 e^{2} / h$ conductance anomaly in one-dimensional systems. J. Phys. Condens. Matter 23, 443201 (2011).

15. Iqbal, M. J. et al. Odd and even Kondo effects from emergent localisation in quantum point contacts. Nature 501, 79-83 (2013).

16. Bauer, F. et al. Microscopic origin of the 0.7 -anomaly in quantum point contacts. Nature 501, 73-78 (2013).

17. Micolich, A. Quantum point contacts: Double or nothing? Nat. Phys. 9, 530-531 (2013)

18. Eriksson, M. A. et al. Cryogenic scanning probe characterization of semiconductor nanostructures. Appl. Phys. Lett. 69, 671 (1996).

19. Topinka, M. A. et al. Imaging coherent electron flow from a quantum point contact. Science 289, 2323 (2000).

20. Topinka, M. A. et al. Coherent branched flow in a two-dimensional electron gas. Nature 410, 183 (2001).

21. LeRoy, B. J. et al. Imaging electron interferometer. Phys. Rev. Lett. 94, 126801 (2005). 
22. Jura, M. P. et al. Unexpected features of branched flow through high-mobility two-dimensional electron gases. Nat. Phys. 3, 841 (2007).

23. Kozikov, A. A. et al. Interference of electrons in backscattering through a quantum point contact. New J. Phys. 15, 013056 (2013).

24. Freyn, A. et al. Scanning gate microscopy of a nanostructure where electrons interact. Phys. Rev. Lett. 100, 226802 (2008).

25. Jura, M. P. et al. Spatially probed electron-electron scattering in a twodimensional electron gas. Phys. Rev. B 82, 155328 (2010).

26. Crook, R. et al. Conductance quantization at a half-integer plateau in a symmetric GaAs quantum wire. Science 312, 1359 (2006).

27. Iagallo, A. et al. Scanning gate imaging of quantum point contacts and the origin of the 0.7 anomaly. Preprint at: http://arXiv:1311.6303v1 (2013).

28. Wigner, E. On the interaction of electrons in metals. Phys. Rev. 46, 1002 (1934).

29. Schulz, H. J. Wigner crystal in one dimension. Phys. Rev. Lett. 71, 1864 (1993).

30. Deshpande, V. V. \& Bockrath, M. The one-dimensional Wigner crystal in carbon nanotubes. Nat. Phys. 4, 314 (2008).

31. Hew, W. K. Incipient formation of an electron lattice in a weakly confined quantum wire. Phys. Rev. Lett. 102, 056804 (2009).

32. Hackens, B. et al. Imaging Coulomb islands in a quantum Hall interferometer. Nat. Commun. 1, 39 (2010).

33. Patel, N. K. et al. Evolution of half plateaus as a function of electric field in a ballistic quasi-one-dimensional constriction. Phys. Rev. B 44, 13549 (1991).

34. Thomas, K. J. et al. Interaction effects in a one-dimensional constriction. Phys. Rev. B 58, 4846 (1998).

35. Komijani, Y. et al. Origins of conductance anomalies in a p-type GaAs quantum point contact. Phys. Rev. B 87, 245406 (2013).

36. Jura, M. P. et al. Electron interferometer formed with a scanning probe tip and quantum point contact. Phys. Rev. B 80, 041303(R) (2009)

37. Georges, A. \& Meir, Y. Electronic correlations in transport through coupled quantum dots. Phys. Rev. Lett. 82, 17 (1999).

38. Aguado, R. \& Langreth, D. C. Out-of-equilibrium Kondo effect in double quantum dots. Phys. Rev. Lett. 85, 1946 (2000).

39. Lopez, R., Aguado, R. \& Platero, G. Nonequilibrium transport through double quantum dots: Kondo effect versus antiferromagnetic coupling. Phys. Rev. Lett. 89, 136802 (2002)

40. Kiselev, M. N., Kikoin, K. \& Molenkamp, L. W. Resonance Kondo tunneling through a double quantum dot at finite bias. Phys. Rev. B 68, 155323 (2003)

41. Sasaki, S. et al. Kondo effect in an integer-spin quantum dot. Nature 405, 764 (2000).

42. Jeong, H., Chang, A. M. \& Melloch, M. R. The Kondo effect in an artificial quantum dot molecule. Science 293, 2221 (2001).

43. Roch, N., Florens, S., Bouchiat, V., Wernsdorfer, W. \& Balestro, F. Quantum phase transition in a single-molecule quantum dot. Nature 453, 633 (2008).

44. Nixon, J. A., Davies, J. H. \& Baranger, H. U. Breakdown of quantized conductance in point contacts calculated using realistic potentials. Phys. Rev. B 43, 12638 (1991).

45. Staring, A. A. M., van Houten, H., Beenakker, C. W. J. \& Foxon, C. T. Coulomb-blockade oscillations in disordered quantum wires. Phys. Rev. B 45, 9222 (1992).

46. Sushkov, O. P. Restricted and unrestricted Hartree-Fock calculations of conductance for a quantum point contact. Phys. Rev. B 67, 195318 (2003).

47. Guçlu, A. D., Umrigar, C. J., Jiang, H. \& Baranger, H. U. Localization in an inhomogeneous quantum wire. Phys. Rev. B 80, 201302(R) (2009).
48. Shulenburger, L., Casula, M., Senatore, G. \& Martin, R. M. Correlation effects in quasi-one-dimensional quantum wires. Phys. Rev. B 78, 165303 (2008).

49. Söffing, S. A. et al. Wigner crystal versus Friedel oscillations in the onedimensional Hubbard model. Phys. Rev. B 79, 195114 (2009).

50. Goldhaber-Gordon, D. et al. Kondo effect in a single-electron transistor. Nature 391, 156 (1998).

51. Iqbal, M. J., de Jong, J. P., Reuter, D., Wieck, A. D. \& van der Wal, C. H Split-gate quantum point contacts with tunable channel length. J. Appl. Phys. 113, 024507 (2013).

52. Davies, J. H., Larkin, I. A. \& Sukhorukov, E. V. Modeling the patterned two dimensional electron gas: electrostatics. J. Appl. Phys. 77, 4504 (1995).

53. Meyer, J. S., Matveev, K. A. \& Larkin, A. I. Transition from a one-dimensional to a quasi-one-dimensional state in interacting quantum wires. Phys. Rev. Lett. 98, 126404 (2007)

54. Smith, L. W. et al. Row coupling in an interacting quasi-one-dimensional quantum wire investigated using transport measurements. Phys. Rev. B 80, 041306(R) (2009)

55. Martins, F. et al. Coherent tunneling accross a quantum point contact in the quantum Hall regime. Sci. Rep. 3, 1416 (2013).

56. Martins, F. et al. Scanning gate spectroscopy of transport across a quantum Hall nano-island. New J. Phys. 15, 013049 (2013).

\section{Acknowledgements}

We thank H. Baranger, J. Meyer, X. Waintal, D. Weinmann, J.-L. Pichard and S. Floren for discussions. This work was supported by the French Agence Nationale de la Recherche ('ITEM-exp' project), by FRFC grant number 2.4503.12, and by FRS-FNRS grants numbers 1.5.044.07.F and J.0067.13. F.M. and B.H. acknowledge support from the Belgian FRS-FNRS, S.F. received support from the FSR at UCL and V.B. acknowledges the award of a 'chair d'excellence' by the Nanosciences foundation in Grenoble.

\section{Author contributions}

B.B. and F.M. performed the low-temperature SGM experiment with the assistance of S.F., B.H. and V.B.; B.B. and H.S. analysed the experimental data and wrote the paper; A.C., A.O. and U.G. grew the GaAs/AlGaAs heterostructure; C.U. and D.M. processed the sample; S.F., B.H. and F.M. built the low-temperature scanning gate microscope; G.B performed the electrostatic simulations; B.B., F.M., S.F., B.H., U.G., D.M., S.H., G.B, V.B. M.S. and H.S. contributed to the conception of the experiment; all authors discussed the results and commented on the manuscript.

\section{Additional information}

Supplementary Information accompanies this paper at http://www.nature.com/ naturecommunications

Competing financial interests: The authors declare no competing financial interests.

Reprints and permission information is available online at http://npg.nature.com/ reprintsandpermissions/

How to cite this article: Brun, B. et al. Wigner and Kondo physics in quantum point contacts revealed by scanning gate microscopy. Nat. Commun. 5:4290 doi: 10.1038/ ncomms5290 (2014) 